Running Head: NEGATIVE AFFECT AND LEARNING ABNORMALITIES

\title{
Anxiety and Depression Related Abnormalities in Socio-affective Learning
} Dylan Hammond ${ }^{1}$, Pengfei $\mathrm{Xu}^{2-4}$, Hui $\mathrm{Ai}^{5}, \&$ Nicholas T. Van Dam ${ }^{1 *}$

${ }^{1}$ Melbourne School of Psychological Sciences, The University of Melbourne, Melbourne, Australia

${ }^{2}$ Beijing Key Laboratory of Applied Experimental Psychology, Faculty of Psychology, Beijing Normal University, Beijing, China

${ }^{3}$ Center for Neuroimaging, Shenzhen Institute of Neuroscience, Shenzhen, China

${ }^{4}$ Great Bay Neuroscience and Technology Research Institute (Hong Kong), Kwun Tong, Hong Kong, China

${ }^{5}$ Shenzhen Key Laboratory of Affective and Social Neuroscience, Shenzhen University, Shenzhen, China

\author{
Author Note \\ Pengfei Xu https://orcid.org/0000-0002-1340-8852 \\ Hui Ai https://orcid.org/0000-0002-3712-1223
}

Nicholas T. Van Dam https://orcid.org/0000-0002-1131-0739

Data and analytic scripts are available via https:/osf.io/quy76/. A preprint of the article is available via https://psyarxiv.com/tg3ec/. We have no conflicts of interest to declare. Dr. Pengfei $\mathrm{Xu}$ acknowledges funding support from National Natural Science Foundation of China (31871137), Young Elite Scientists Sponsorship Program by China Association for Science and 
NEGATIVE AFFECT AND LEARNING ABNORMALITIES 2

Technology (YESS20180158), and Shenzhen Science and Technology Research Funding

Program (JCYJ20180507183500566).

Correspondence concerning this article should be addressed to Dr. Nicholas T. Van Dam, Melbourne School of Psychological Sciences, The University of Melbourne, Parkville VIC Australia, 3010. E-mail: nicholas.vandam@unimelb.edu.au 
NEGATIVE AFFECT AND LEARNING ABNORMALITIES 3

\begin{abstract}
Affective distress (as observed in anxiety and depression) has been observed to be related to insufficient sensitivity to changing reinforcement during operant learning. Whether such findings are specific to anxiety or depression is unclear given a wider literature relating negative affect to abnormal learning and the possibility that relationships are not consistent across incentive types (i.e. punishment and reward) and outcomes (i.e., positive or negative). In two separate samples (convenience $n=76$; psychiatrically assessed $n=49$ ), participants completed an operant learning task with positive, negative, and neutral socio-affective feedback, designed to assess adaptive responses to changing environmental volatility. General affective distress, rather than anxiety or depression specifically, was related to an increase in the rate of learning for negative (i.e., worse-than-expected) outcomes in volatile, relative to stable, environments. Our results suggest an important but general role of affective distress in overweighting negative feedback when the value of an action becomes uncertain, as when environmental volatility increases.

Keywords: anxiety, depression, learning, social feedback, reinforcement-learning, computational psychiatry
\end{abstract}


NEGATIVE AFFECT AND LEARNING ABNORMALITIES 4

\section{Introduction}

Human behaviour is guided by the simultaneous goals of maximising reward while minimising punishment. However, individuals with depression and anxiety often exhibit behaviours at odds with their best interests (Browning et al., 2015; Gagne et al., 2020). Seminal work by Browning and colleagues (2015) suggests that anxiety is associated with impaired learning in aversive environments, potentially explaining maladaptive behaviour as the consequence of abnormal learning. Learning abnormalities have also been shown in depression (Robinson \& Chase, 2017), and recent work shows associations with general affective distress (Gagne et al., 2020), raising questions about the specificity of learning deficits to anxiety (Pulcu \& Browning, 2019). Concerns about the overlap in symptom features of anxiety and depression (Regier et al., 2013; Watson, 2009), as well as a focus on threat learning and reward learning in anxiety and depression respectively (see Bishop \& Gagne, 2018), raise questions as to whether findings such as those identified by Browning and colleagues (2015) may relate more generally to negative affect rather than anxiety specifically, as suggested by more recent work (Gagne et al., 2020).

Utilising recent advances in the computational modelling of behaviour, researchers have aimed to identify behavioural differences in attention and learning that may underpin a variety of mental disorders (Bishop \& Gagne, 2018; Montague et al., 2012). Computational modelling permits isolation of specific processes from broader behaviours; isolated components can then be subjected to various algorithms to explore associations with meaningful clinical presentations and outcomes (Adams et al. 2016; Wiecki et al). By identifying and quantifying component mechanisms most relevant to a given clinical presentation, modelling in the context of psychopathology promises to move the field towards personalisation, providing potential phenotypes and/or markers that could be meaningful on a smaller scale (Adams et al., 2016; 
NEGATIVE AFFECT AND LEARNING ABNORMALITIES 5

Wiecki et al., 2015), especially in the context of anxiety and depression (see Regier et al. 2012; Watson, 2009).

While various abnormalities in attention and learning have been emphasized with respect to anxiety and/or depression, there is a notable tendency in the scientific literature to examine these disorders separately (Bishop \& Gagne, 2018). Bishop and Gagne (2018) suggest that the field emphasises abnormalities in reward processing in relation to depression and abnormalities in threat/punishment processing in relation to anxiety. As the authors acknowledge, it is unclear whether such associations reflect the reality of clinical presentations or simply what has been most commonly studied. Consequently, a significant barrier to the characterisation of these conditions is the continued study of each disorder in isolation, often using different learning models (i.e., associative vs. operant) and mixed stimuli (i.e., electrical shock and money).

Recent findings have shown that a general, internalizing factor (rather than anxiety or depression specifically) was associated with poorer overall learning and decreased learning in better-than-expected outcomes under volatile environments (Gagne et al., 2020). However, Gagne and colleagues (2020), as is consistent with most work in the field, compared nonequivalent outcomes across both versions of their tasks (i.e., positive reinforcement - monetary gain vs. negative punishment - monetary loss from previously provided funds; positive punishment - electrical shock vs. positive reinforcement - monetary gain). Other variants, such as those used by Browning and colleagues (2015) focus on punishment-minimisation (shock vs. nothing) or reward-maximization (monetary gain vs. nothing). While common lore suggests a similar underlying neural basis to value-based decision-making processes (Clithero \& Rangel, 2014), the underlying neural associates seem to be different when feedback modality and incentive condition are not confounded (Pauli et al., 2015). Socio-affective feedback, functioning as a positive reinforcer of similar scale in both contexts, has the potential to rectify this issue and 


\section{NEGATIVE AFFECT AND LEARNING ABNORMALITIES 6}

render results from different incentive conditions more comparable. To address the concerns raised by previous works, we utilised socio-affective feedback as reinforcement stimuli to equate feedback across varying, positive and negative incentive conditions while measuring anxiety and depression among separate clinical and nonclinical samples. We also examined paradigms specific to reward-maximisation and punishment-minimisation to examine whether one approach may have strong associations to measures of affective distress.

Previous work has found that participants are generally able to optimally adapt their learning to varying reinforcement conditinos (Behrens et al., 2007; Pulcu \& Browning, 2017) and that they are able to do so using social information (Behrens et al., 2008). However, (Browning et al., 2015) found that those with high trait anxiety ( $\mathrm{n}=31$ total) struggled to adjust their learning under punishment-minimisation when outcomes were potentially aversive, leading to poorer performance in the task and suggesting that these individuals may not be able to accurately estimate environmental volatility. Importantly, this offers a potentially causal explanation of trait anxiety as the result of a learning deficit, since a deficit in estimating environmental volatility will likely lead to over-estimation of irreducible uncertainty, subjective estimates of which correlate highly with subjective and physiological stress (De Berker et al., 2016).

While the results of Browning and colleagues (2015) were explicitly tied to trait anxiety, learning abnormalities may be a general property of the higher negative affect of emotional disorders (cf. (Watson, 2009). The association between trait anxiety and maladaptive learning was evident only in conditions of punishment-minimisation (Browning et al. 2015). When the incentive condition was changed to that of reward-maximisation, the association with trait anxiety was not significant. In more recent work, Gagne and colleagues (2020) found impoverished learning adaptation under volatility associates with general affective distress rather than anxiety or depression specifically, using a number of different measures of anxiety and 


\section{NEGATIVE AFFECT AND LEARNING ABNORMALITIES 7}

depression. However, the tasks used directly compared reward to punishment which can make it difficult to disentangle incentive from feedback condition.

Given the association of depression and anxiety (see e.g., Watson, 2009), it is important to consider what is known about learning in depression as well. Previous literature relating depression to performance under varying incentive conditions tends to show an inverse pattern of results to those discussed above (maladaptive learning for reward-maximisation; see e.g., (Beevers et al., 2013; Maddox et al., 2012). For example, researchers have found that depressed individuals across two studies performed better and had higher learning-rates under conditions of punishment-minimisation relative both to healthy controls and their own performance under reward-maximisation (Beevers et al., 2013; Maddox et al., 2012), suggesting improved punishment learning. Other studies have found that those with clinically significant depression struggle to learn at all under conditions of reward-maximisation (DelDonno et al., 2015; Herzallah et al., 2013) and are overly responsive to aversive feedback even when this feedback is misleading (Murphy et al., 2003; Tavares et al., 2008). The work of Gagne and colleagues (2020) observed the most pronounced effects when those with elevated affective distress received betterthan-expected outcomes (i.e., pessimistic bias) under volatile environments, displaying a decrease in learning.

In order to assess whether abnormal volatility-based learning is specific (a) to anxiety and (b) to aversive environments, participants from two sites completed a modified version of the previous task (Behrens et al. 2007; Browning et al. 2015) along with several self-report measures related to anxiety and depression. Additionally, participants in one sample were recruited with an emphasis on current or historic clinically significant affective disorder and underwent a semistructured psychiatric assessment. Given the findings of prior research, we expected that (i) learning-rate adaptation would be negatively related to measures of anxiety and (ii) measures of 
depression would be related to the same effect, albeit to a reduced extent, as well as (iii) reduced learning overall.

\section{Methods}

\section{Participants}

We collected data from two sites to examine the robustness of effects and explore patterns spanning a subclinical through clinical spectrum.

Sample 1 (Non-Clinical). At the first site (Shenzhen University), we focused exclusively on collecting data in a non-clinical sample, excluding those with a current or historic psychiatric or neurological diagnosis. Participants were 100 Chinese-speaking undergraduate students.

Sample 2 (Clinical). At the second site (University of Melbourne), we focused on individuals with mild-moderate symptoms of anxiety and/or depression, deliberately recruiting those known to have either current or historic problems with mental health, as well as those with non-clinical symptoms. Participants were 95 individuals, aged between 18 and 44 years, participating in a one-year, prospective longitudinal study tracking anxious and depressive symptoms. Participants were required to have sufficient comprehension of English to complete measures and mild to moderate symptoms of psychological distress, as indicated by K10 scores (Kessler et al., 2002) between 15 and 39 (Andrews \& Slade, 2001). Additional exclusion criteria comprised: (1) current neurological or chronic medical condition, (2) current or historic serious mental illness (i.e. a psychotic disorder, a bipolar affective disorder, or treatment resistant depression) and/or a substance use disorder (excepting mild - moderate alcohol, marijuana, and nicotine use disorders).

\section{Procedure}

Sample 1. Once determined to be eligible, all participants provided written informed consent before completing self-report measures and an operant learning task, modelled on work 
NEGATIVE AFFECT AND LEARNING ABNORMALITIES 9

by Behrens and colleagues (2007) and Browning and colleagues (2015), and described in more detail below. Individuals were reimbursed 45-60 RMB for their time. Work in Shenzhen was approved by the local ethics committee.

Sample 2. Potential participants were pre-screened via an online battery of assessments to examine inclusion/exclusion criteria. Upon determination of eligibility, potential participants attended a laboratory session where they provided written informed consent, completed a semistructured psychiatric interview, the operant learning task, a variation of the Iowa Gambling Task (IGT), and several self-report measures. All participants were assessed for psychiatric disorders via the Mini International Neuropsychiatric Interview (MINI) version 7.0.2 for DSM-5 (Sheehan et al., 1998), administered by provisional psychologists under the supervision of the senior author. Any participants deemed ineligible after the interview were reimbursed 20 AUD for their time and discharged. Those participants deemed eligible went on to complete experimental tasks and self-report measures, receiving an additional reimbursement of 40 AUD upon completion of these tasks. While participants went on to complete weekly assessments over a period of one year, only those measures relevant to the present analysis are described below. All procedures were approved by the local ethics committee.

\section{Experimental Task}

The operant learning task modelled on prior work (Behrens et al., 2007, 2008; Browning et al., 2015; Pulcu \& Browning, 2017), was programmed and executed using PsychoPy2 version 1.90.3 (Peirce et al., 2019). It comprised two blocks of 120 trials each, in which participants were required to learn, by trial and error, which of two options led to the most positive/least negative feedback (see Figure 1). Stimuli were colour unique 'slot machine' images. Feedback was socioaffective, comprising facial images and short written expressions. All participants completed two versions of the task in counterbalanced order, with incentive condition varying across sessions. In 
addition to varying incentives, environmental volatility varied across each block of the task with feedback patterns following those presented in previous work (Behrens et al., 2007, 2008; Browning et al., 2015; Pulcu \& Browning, 2017). On-screen stimuli were probabilistically associated with a particular outcome (positive/negative).

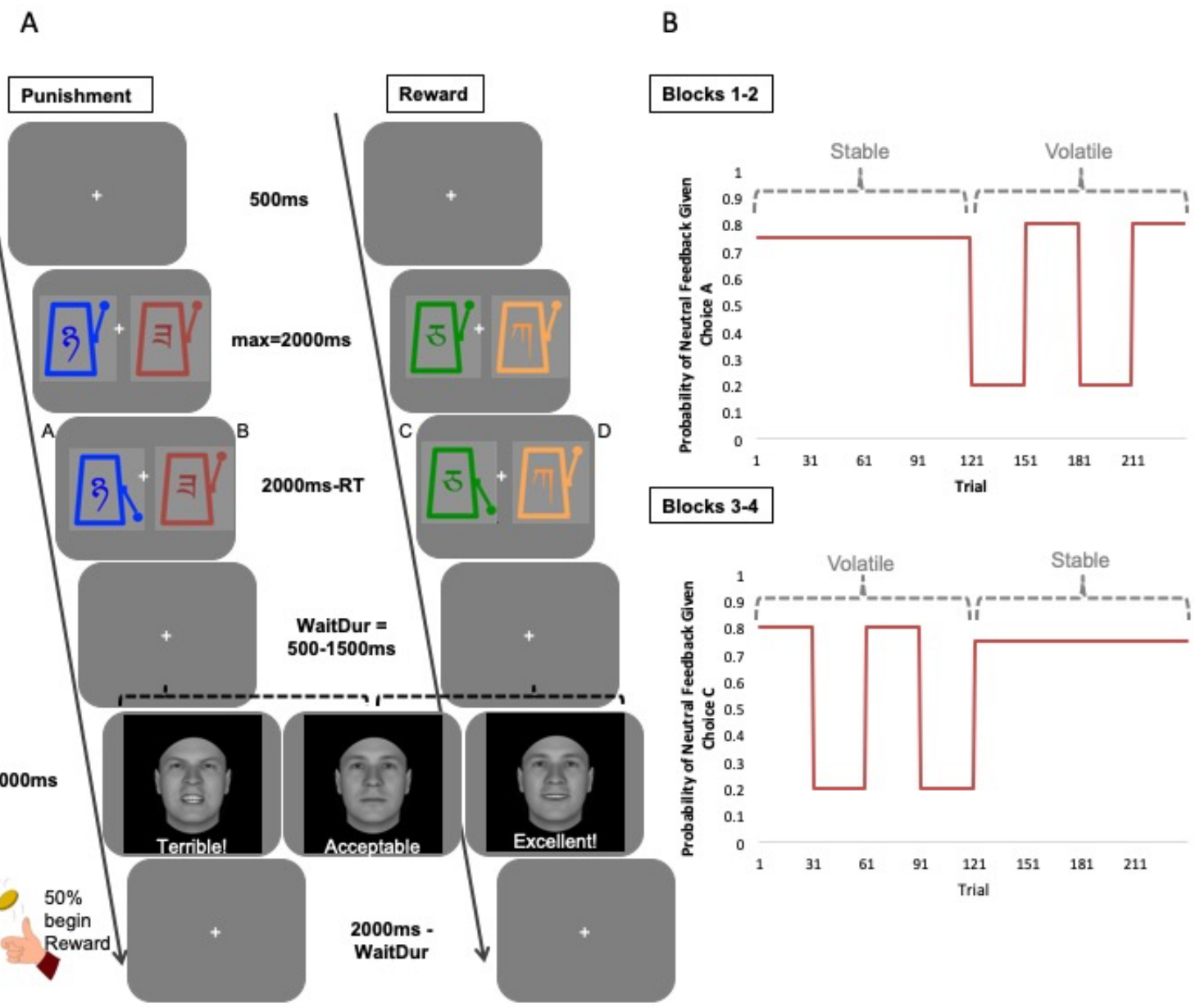

Figure 1. Schematic of Task Structure. Participants completed two separate versions of the task, (1) rewardmaximisatiom: neutral and positive feedback and (2) punishment-minimisation: neutral and negative feedback. (A) Schematic of a trial. Participants were presented with two coloured 'slot machines' and asked to choose one. On each trial, one option resulted in a 'win' (a happy face under reward-maximisation or a neutral face under punishmentminimisation) and the other a 'loss' (a neutral face under reward-maximisation an angry face under punishmentminimisation). Each option was associated probabilistically with a particular outcome. B) Each version of the task comprised two blocks of 120 trials each. Environmental volatility varied across blocks. During stable blocks, the 


\section{NEGATIVE AFFECT AND LEARNING ABNORMALITIES 11}

reinforcement probability remained constant. During volatile blocks, choice-outcome contingencies changed every 30 trials.

During stable blocks, the reinforcement probability remained constant for the entire 120 trials. During volatile blocks, choice-outcome contingencies changed every 30 trials (see Figure 1B). The order of presentation of volatility block was counter-balanced across participants and each version of the task took approximately 15 minutes to complete.

On each trial, participants were presented with a fixation cross flanked on either side by choice stimuli. Participants had 2000ms to respond, else both options disappeared and participants were instructed to "Please respond quickly". Following a response, the image changed slightly to indicate a choice had been registered. Slot-machines remained onscreen for 2000ms minus the participant's reaction time. Subsequently, a fixation cross was presented for 500 to $2000 \mathrm{~ms}$ (jittered). Participants were presented with socio-affective feedback for $1500 \mathrm{~ms}$ before a central fixation cross was presented for another jittered interval equal to $2000 \mathrm{~ms}$ minus the initial interval's length. In addition to the 120 task trials in each block, participants also completed 4 catch trials designed to identify careless responding. On catch trials, participants were required to correctly identify the location (i.e., left or right) of a black 'slot machine'. Correct identification resulted in neutral feedback while incorrect identification resulted in an instruction to "Please pay attention".

Task Stimuli. Visual stimuli were 'slot machines', coloured according to incentive condition: In the reward condition, slot-machines were green and orange, while in the punishment condition, slot-machines were blue and red. Eighteen separate angry, happy, and neutral forwardfacing, computer generated facial images were selected from the Bochum Emotional Stimulus Set (BESST; (Thoma et al., 2013) for feedback. Images were selected on the basis of emotional classification (classification rate: $M=0.92, S D=0.08$ ), as well as perceived realness on a 5-point 
scale $(M=3.78, S D=0.62)$. Prior to use, images were converted to grey scale and the periphery of the face (where hair would appear but was absent) were masked to increase the perception of realness. Each face was then paired with a written exclamation, appropriate to their intended incentive purpose (e.g., "Terrific" for a happy face, "ok" for a neutral face, and "Terrible!" for an angry face).

\section{Self-Report Measures (Sample 1)}

Self-rating Depression Scale (SDS). The SDS (Zung, 1965) is a 20-item self-report measure requiring participants to endorse statements on a 4-point scale ranging from "none/a little of the time" to "most/all of the time". The C-SDS has been shown to possess adequate reliability in a sample similar to our own (test-retest $=.83$; (Liu et al., 1999). The measure has also been shown to possess good validity, as well as reliability, in other samples (Thurber et al., 2002).

State Trait Anxiety Inventory (STAI). The STAI (Spielberger et al., 1983) is a 20-item self-report measure requiring participants to rate how they generally feel, on a 4-point scale. The STAI has been shown to possess adequate reliability $(\alpha=.81$; (Shek, 1988) and validity (Shek, 1993). The STAI is known to conflate items measuring both anxiety and depression (Bieling et al., 1998). Consequently, we also calculated separate STAI anxiety and depression scores using the factor structure indicated by (Bieling et al., 1998).

\section{Self-Report Measures (Sample 2)}

K10. The K10 (Kessler et al., 2002) is a 10-item measure assessing symptoms of psychological distress over the past month. Participants answer questions on a five-point scale from $1=$ "None of the time" to $5=$ "All of the time". Scores of 20 or higher typically indicate presence of a mental disorder (Kessler et al., 2002). Previous work has shown that $67.5 \%$ of 
respondents exhibit scores of $10-14$, while only $0.4 \%$ of individuals exhibit scores $>39$ (Andrews \& Slade, 2001).

Mini International Neuropsychiatric Interview (MINI). The MINI (Sheehan et al., 1998) is a semi-structured clinical interview designed to provide fast and accurate diagnoses using criteria from the Diagnostic and Statistical Manual for Mental Disorders (DSM; (American Psychiatric Association, 2013). Version 5.0 of the interview has been validated against the Composite International Diagnostic Interview (CIDI; (Robins et al., 1988) and Structured Clinical Interview for DSM-III (SCID-III; (Spitzer et al., 1988), demonstrating comparable performance to these two gold-standard tools for psychiatric classification (Lecrubier et al., 1997). Version 7.0.2 of the MINI has been updated to reflect changes to diagnostic criteria.

Depression, Anxiety, and Stress Scales (DASS-42). The DASS-42 (P. F. Lovibond \& Lovibond, 1995) is a 42 item self-report instrument designed to measure three key dimensions of distress: depression, anxiety, and stress. Each item requires participants to reflect on the previous week using a 4-point scale from $0=$ "Did not apply to me at all" to 3 = "Applied to me very much or most of the time". Each subscale of the DASS-42 possesses moderate reliability (testretest $=.71-.81 ;($ Brown et al., 1997) and good internal consistency $(\alpha=.92-.97 ;$ (Antony et al., 1998). Additionally, the three-factor model of the DASS-42 has been supported by several exploratory and confirmatory factor analyses (Antony et al., 1998; Brown et al., 1997; P. F. Lovibond \& Lovibond, 1995).

\section{Computational Modelling}

Models. We fit two model variants to choice $[A, B]$ and outcome $[-1,1]$ data from each 120-trial block of the operant learning task. Choice behaviour was modelled using a softmax action-selector as follows (Equation 1):

1 .

$$
P(A)_{t}=\frac{1}{\left.1+e^{-\beta(E V[A]} t^{-E V[B]} t\right)}
$$


NEGATIVE AFFECT AND LEARNING ABNORMALITIES 14

$P(A)$ is the probability of choosing option $A$ on trial $t, E V[A]$ is the expected value of option $A$ on trial $t$, and $\beta$ is the inverse temperature, controlling the degree to which expected values determine choice behaviour (high values lead to more deterministic behaviour; see Figure 8). Expected values for both options were initialised at zero and updated using a delta updating rule as specified in the following equations (Equation $2 \& 3$ ):

2.

$$
P E_{t}=r_{t}-E V[c]_{t}
$$

3.

$$
E V[c]_{t+1}=E V[c]_{t}+\alpha P E_{t}
$$

$P E$ is the prediction error for trial $t, r$ is the outcome on trial $t, E V[c]$ is the expected value for the chosen option on trial $t$, and $\alpha$ is the learning-rate (between 0 and 1), controlling the degree to which current prediction errors are used to determine future expected values. Higher learning-rates lead to a greater correspondence between current prediction errors and subsequent expected values, with $E V[c]_{t+1}$ approaching $P E_{t}$ as $\alpha$ approaches one. The second model variant utilised two distinct learning-rates, one for positive and another for negative prediction errors such that Equation 3 becomes:

4.

$$
E V[c]_{t+1}=\left\{\begin{array}{l}
E V[c]_{t}+\alpha_{p o s} P E_{t}, P E_{t}>0 \\
E V[c]_{t}+\alpha_{n e g} P E_{t}, P E_{t} \leq 0
\end{array}\right.
$$

Since in our experiment outcomes were dichotomous (e.g., either 0 or 1), prediction error was confounded with outcome (e.g., under reward-maximisation, a happy face will always result in a positive prediction error and a neutral face will always result in a negative prediction error). For this reason, we have used the term 'outcome' in place of 'prediction-error' in the following text. Despite this, the reader should note that the model used is unable to distinguish between the effects of outcome and prediction-error.

Parameter Estimation. Models were fit to behavioural data using a combination of JAGS (Denwood, 2016), Stan (Carpenter et al., 2017), and the hBayesDM package (Ahn et al., 2017) in 
R version 3.5.3 (Team, 2013). Hierarchical Bayesian analyses (HBA) differ from individual estimation methods in that group level parameters, known as hyper-parameters, both inform and are informed by the performance of individuals. A consequence of this, known as shrinkage, is that individual parameter estimates tend to get pulled towards the mean of group performance, relative to estimates based on individual performance alone. HBA are particularly useful when the number of trials per participant is low, as is often the case in applications where participant fatigue is a concern.

Because individual parameter estimates derived using HBA will depend on each other, we included in the HBA only those individuals who demonstrated that they were learning in the task according to instructions. To assess task compliance, we first fit both models to the subject-level data, using JAGS in R, and compared their performance, using the Bayesian Information Criterion (BIC), to a random-choice model. If, on any block, the random-choice model was determined to be the most parsimonious explanation of behaviour, this block was not included in the HBA. Excluding data which the model cannot adequately describe is recommended practice (Hitchcock et al., 2017), since point estimates of parameters in these instances will be meaningless. The above process meant that some participants contributed to the HBA even though they were excluded from the final analysis. We felt that this achieved a balance between excluding poor quality data from final analysis while including informative data which may contribute to more reliable individual parameter estimates through their effect on group level parameters.

Model Selection. Following previous research, we selected between model variants [dualDelta - separate learning-rates for negative and positive outcomes vs. delta - single learningrate] by fitting both models to the data, estimating separate sets of hyper-parameters for each sample and volatility by incentive condition: punishment-minimisation stable, punishment- 
minimisation volatile, reward-maximisation stable, and reward-maximisation volatile (see Table 2). The best fitting model was defined as the model with the lowest Leave-One-Out Information Criterion (LOOIC; (Vehtari et al., 2017) summed over four conditions and two samples. Subsequent to initial model selection, we tested how well the best fitting model performed in terms of model fit as we reduced the number of hyper-parameters, exploring four different combinations: (1) separate sets of hyper-parameters for each incentive by volatility condition, (2) separate sets of hyper-parameters for each incentive condition only, (3) separate sets of hyperparameters for each volatility condition only, and (4) a single set of hyper-parameters for all conditions (see Table 2). Priors used for individual and group level parameters were the weakly informative default priors in hBaysDM (Ahn et al., 2017).

\section{Results}

\section{Participants}

100 participants from Sample 1 (non-clinical) and 95 from Sample 2 (clinical) completed the task (Figure 1). Participants who completed all blocks and provided variable response patterns not best explained by a guessing model were included in the final analysis. Those excluded due to task performance were not significantly different from those retained on measures of anxiety or depression (see Supplemental Materials). Final samples were 76 (Sample 1) and 49 (Sample 2; see Table 1) after exclusions were applied. In the first sample, participant STAI-T scores were higher than (a) cut-off scores indicative of anxiety disorders (Hishinuma et al., 2001) and (b) normative data from similarly-aged individuals (Knight et al., 1983). Levels of depression, as measured by SDS, were similar to those of normative, rather than clinical, groups (Knight et al., 1983). Among the second sample, approximately $44.9 \%$ of participants met criteria for a current psychiatric disorder using the MINI, most with Generalized Anxiety Disorder (GAD only: 26.5\%, $\mathrm{n}=13$ ) or Major Depressive Disorder (MDD only: $8.2 \%$, 
$\mathrm{n}=4$; GAD \& MDD: $6.1 \%, \mathrm{n}=3)$. The majority $(65 \%)$ exhibited evidence of a lifetime psychiatric diagnosis. Additionally, the sample was, on average, mildly anxious, mildly depressed, and mildly stressed (per recommended cut-off scores, see (S. H. Lovibond \& Lovibond, 1996), though all distributions were positively skewed with $30.6 \%(n=15)$ and $42.9 \%(n=21)$ in moderate or greater categories for depression and anxiety, respectively (see Supplemental

\section{Material).}

Table 1. Means and standard deviations for demographic and symptom information.

\begin{tabular}{lll}
\hline & Sample 1 & Sample 2 \\
Variable & $M(S D)$ & $M(S D)$ \\
\hline Age & $20.09(2.56)$ & $23.33(4.51)$ \\
Gender & $43.42 \%$ Female & $79.59 \%$ Female \\
STAI-T Tot & $42.30(8.46)$ & - \\
STAI-T Anx & $12.62(3.57)$ & - \\
STAI-T-Dep & $29.68(5.56)$ & - \\
SDS & $35.97(7.02)$ & - \\
DASS-Dep & - & $9.78(9.48)$ \\
DASS-Anx & - & $7.37(6.62)$ \\
DASS-Str & - & $9.78(8.51)$ \\
DASS-Tot & - & $28.73(22.09)$ \\
\hline
\end{tabular}

STAI-T = State Trait Anxiety Inventory, Trait scale; - Anx = Set of items previously identified by (Bieling et al., 1998) to be primarily related to anxiety; -Dep = Set of items previously identified to be primarily related to depression; SDS = Self-rating Depression Scale; DASS = Depression, Anxiety, and Stress Scales.

\section{Model Selection and Estimation}

Comparable to previous findings (Pulcu \& Browning, 2017), the most parsimonious model had separate learning-rates for positive and negative outcomes, a single parameter - the 
inverse temperature - governing choice determinism, and a single set of hyper-parameters for all samples, incentive conditions, and volatility levels (see Table S3). Figures 2 and $\mathbf{3}$ display the distributional properties and summary statistics of parameter estimates from the final model. Additionally, inverse temperature (across all conditions of the task, within subjects) was negatively correlated with learning-rates for negative outcomes in both Sample $1, r_{(302)}=-0.45$ $(95 \% C I=[-.53,-.35])$, and Sample $2, r_{(178)}=-0.65(95 \% C I=[-.73,-.56])$. While parameter correlations can be a sign of parameter redundancy (i.e., the parameters are not independent and may have compensatory effects), no such correlations were observed when the model was fit to simulated data (see Supplementary Materials). Consequently, the high correlations found in our sample are likely a property of the data, rather than the model itself, suggesting that those with lower learning-rates tended to be more consistent responders.

\section{Learning, incentive conditions, environmental volatility and outcome valence}

We assessed the effects of incentive condition, volatility, and outcome valence on learning-rates using separate within-subjects ANOVAs for each sample. Bonett's $\delta$ ((Bonett, 2008) was employed as a robust measure of effect size. Additionally, we included incentive order as a between-subjects factor to assess whether presentation order influenced response patterns (see Table 2 for test results). Finally, since learning-rates were restricted to $0-1$ and the statistical tests assume variation on the real number line, raw learning-rates were logit-transformed prior to statistical analyses.

Sample 1 (Non-Clinical). Consistent with previous work (Behrens et al., 2007, 2008; Pulcu \& Browning, 2017), learning-rates were higher under volatile relative to stable environments $(\delta=0.26)$. Additionally, learning-rates tended to be higher under conditions of punishment-minimisation relative to reward-maximisation $(\delta=0.11)$. While the effect of volatility did not vary by incentive condition (reward vs. punishment), outcome valence did $(\delta=$ 
0.31): In the reward-maximisation condition (neutral and happy faces), people were more affected by positive outcomes (i.e., happy faces) relative to negative outcomes (i.e., neutral faces), while the reverse was true under conditions of punishment-minimisation (angry and neutral faces). Put another way, the significant interaction between incentive condition and outcome valence indicates that people were less affected by neutral faces relative to emotional faces (i.e., neutral and angry faces), irrespective of the incentive condition.

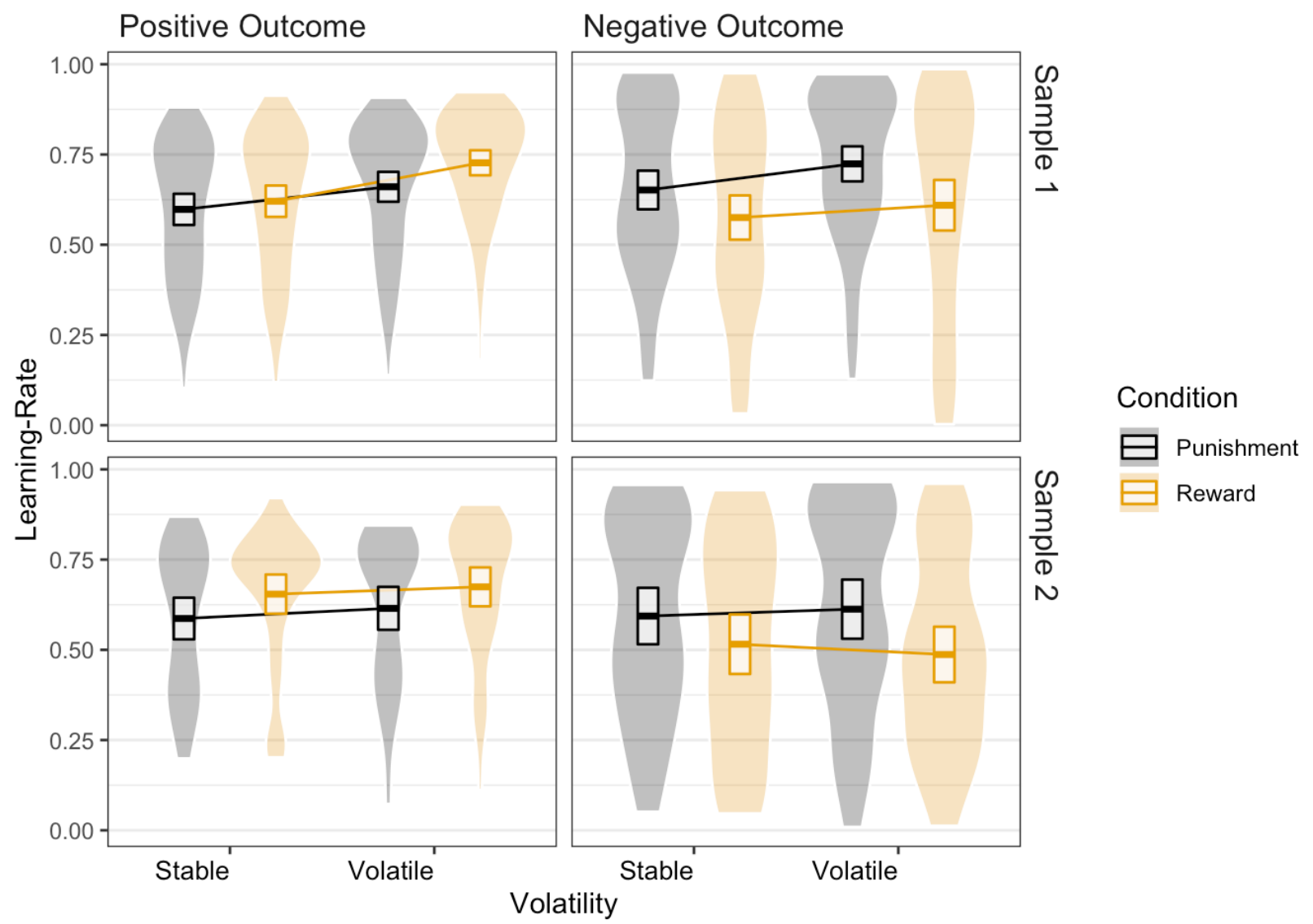

Figure 2. Distribution of learning-rates by volatility, incentive condition, and outcome

valence. Boxes represent one standard error of the mean, with the mean value marked by the mid-line of each box.

Though the order in which participants completed the operant learning task did not affect response to the volatility manipulation, it had effects on behaviour. Learning-rates tended to be higher under punishment-minimisation for those who experienced this condition first, while those 
who experienced reward-maximisation first performed similarly in both conditions $(\delta=0.24)$. Additionally, learning-rates tended to be higher for positive outcomes (i.e., outcome better than expected) relative to negative outcomes (i.e., outcome worse than expected) when punishmentminimisation was presented first, while the reverse was true (higher learning-rate for negative vs. positive outcomes) for those who experienced reward-maximisation first $(\delta=0.26)$. Finally, a significant three-way interaction between incentive condition, outcome valence, and incentive order indicated that, when punishment was presented before reward, people were much less sensitive under conditions of reward-maximisation to negative outcomes (i.e. neutral faces) and were more sensitive than expected in all other instances $(\delta=0.27)$.

Sample 2 (Clinical). Experimental manipulations functioned in markedly different ways in our second sample, collected with an emphasis on psychological distress. The main effect of volatility was not significant, indicating that the experimental manipulation of environmental volatility did not have its intended effect on learning-rate in this sample. Additionally, there was no significant effect of incentive condition nor were there significant relationships between presentation order and learning. The only similarity between the two samples was a significant interaction between incentive condition and outcome valence $(\delta=0.34)$ echoing what was found in the first sample: individuals were more sensitive to socio-emotional feedback relative to neutral feedback.

\section{Choice determinism, incentive condition, and environmental volatility}

Choice determinism - indexed by the inverse-temperature parameter, $\beta$ - was not significantly affected by volatility. However, participants tended to be more consistent under reward-maximisation relative to punishment-minimisation, $\left(\delta=0.23, F_{(1,74)}=6.01, p=.02\right)$ and there were again large effects of session order in Sample 1. In this convenience sample, session order interacted with incentive condition $\left(\delta=-0.55, F_{(1,74)}=35.14, p<.001\right)$; such that while 
participants tended to respond more consistently in their second session relative to their first (a standard effect of task repetition), this was greatly accentuated if reward was experienced before punishment, with participant's responding relatively inconsistently under reward if this was the first condition they experienced. Finally, choice determinism was not related to symptom measures in either sample (see Supplemental Material for details).

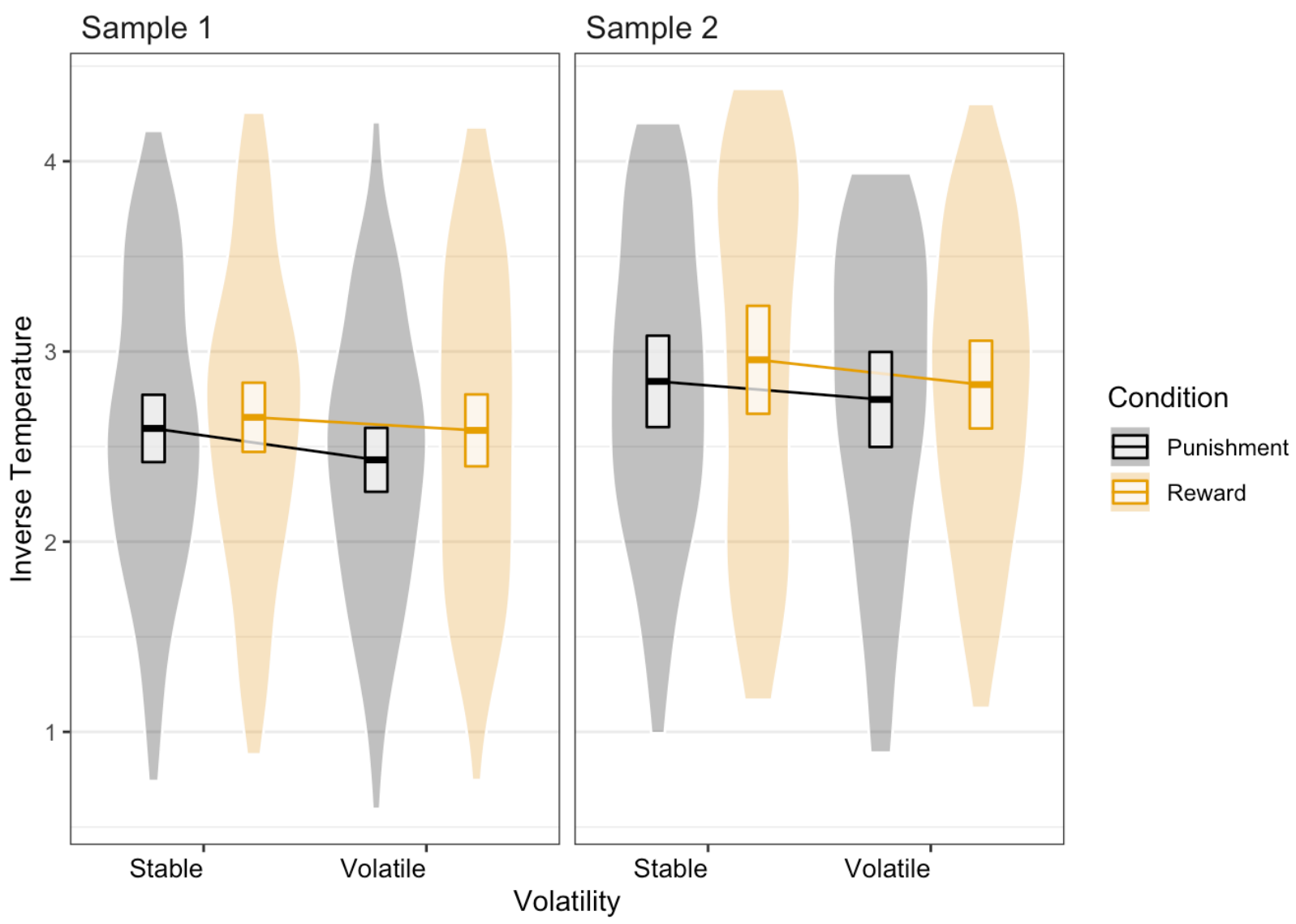

Figure 3. Distribution of inverse-temperatures by volatility and incentive condition. Boxes represent one standard error of the mean, with the mean value marked by the mid-line of each box. 
Table 2. Results of mixed-effects ANOVAS using logistic learning-rate as a dependent variable, incentive condition (punishmentminimisation or reward maximisation), volatility (stable or volatile), and outcome valence (positive or negative) as within-subjects effects, and session order (whether reward or punishment was presented first) as a between-subjects effect.

\begin{tabular}{|c|c|c|c|c|c|c|}
\hline \multirow[b]{2}{*}{ Effect } & \multicolumn{3}{|c|}{ Sample 1} & \multicolumn{3}{|c|}{ Sample 2} \\
\hline & $d f$ & $F$ & $p$ & $d f$ & $F$ & $p$ \\
\hline Order & $(1,74)$ & 0.23 & .63 & $(1,47)$ & 2.47 & .12 \\
\hline Incentive & $(1,74)$ & 7.05 & .01 & $(1,47)$ & 1.17 & .28 \\
\hline Volatility & $(1,74)$ & 21.73 & $<.001$ & $(1,47)$ & 0.15 & .70 \\
\hline Outcome & $(1,74)$ & 0.00 & .97 & $(1,47)$ & 3.85 & .06 \\
\hline Order X Incentive & $(1,74)$ & 12.12 & $<.001$ & $(1,47)$ & 0.02 & .88 \\
\hline Order X Volatility & $(1,74)$ & 1.43 & .24 & $(1,47)$ & 0.13 & .72 \\
\hline Order X Outcome & $(1,74)$ & 6.09 & .02 & $(1,47)$ & 0.29 & .59 \\
\hline Incentive X Volatility & $(1,74)$ & 0.10 & .75 & $(1,47)$ & 0.26 & .61 \\
\hline Incentive X Outcome & $(1,74)$ & 20.33 & $<.001$ & $(1,47)$ & 17.71 & $<.001$ \\
\hline Volatility X Outcome & $(1,74)$ & 1.62 & .21 & $(1,47)$ & 0.65 & .42 \\
\hline Order X Incentive X Volatility & $(1,74)$ & 1.33 & .25 & $(1,47)$ & 1.02 & .32 \\
\hline Order X Incentive X Outcome & $(1,74)$ & 11.40 & .001 & $(1,47)$ & 0.52 & .48 \\
\hline Order X Volatility X Outcome & $(1,74)$ & 1.86 & .18 & $(1,47)$ & 0.19 & .66 \\
\hline Incentive X Volatility X Outcome & $(1,74)$ & 3.88 & .05 & $(1,47)$ & 0.29 & .59 \\
\hline Order X Incentive X Volatility X Outcome & $(1,74)$ & 3.64 & .06 & $(1,47)$ & 0.11 & .74 \\
\hline
\end{tabular}




\section{Emotional disorder and volatility-induced learning-rate adaptation}

To test our primary hypothesis related to the relationship between volatility-induced learning-rate adaptation and symptoms of anxiety/depression, we replicated the approach of Browning and colleagues (2015), calculating Pearson correlations between relative learning-rates and symptoms measures (Table 3 ).

Sample 1 (Non-Clinical). In contrast to the results of (Browning et al., 2015), total STAI$\mathrm{T}$ was not significantly related to volatility-induced learning-rate adaption in either incentive condition (see Table 3). Instead, STAI-T-Anx (cf. (Bieling et al., 1998), along with SDS, were associated with volatility-induced learning-rate adaptations under conditions of punishmentminimization. Additionally, this association depended on the valence of the outcome. While both STAI-T-Anx and SDS were positively correlated with volatility-induced learning-rate adaptation for negative outcomes, the same measures were negatively correlated with learning-rate adaption for positive outcomes, though the latter findings were not significant.

Table 3. Correlations and $95 \%$ confidence intervals between symptom measures and the relative learning-rate (Volatile - Stable).

\begin{tabular}{|c|c|c|c|c|c|}
\hline & & Punishment & & Reward & \\
\hline Measure & $d f$ & Negative & Positive & Negative & Positive \\
\hline SDS & 74 & $.36(.14, .54) *$ & $-.16(-.37, .07)$ & $-.05(-.27, .18)$ & $-.00(-.23, .22)$ \\
\hline STAI-T-Anx & 74 & $.28(.05, .47) *$ & $-.12(-.34, .11)$ & $-.01(-.23, .22)$ & $.00(-.23, .23)$ \\
\hline STAI-T-Dep & 74 & $.11(-.12, .32)$ & $-.08(-.30, .15)$ & $.01(-.21, .24)$ & $-.01(-.24, .21)$ \\
\hline
\end{tabular}




\begin{tabular}{llll|ll} 
STAI-T-Tot & 74 & $.19(-.04, .40)$ & $-.11(-.32, .12)$ & $.01(-.22, .23)$ & $-.01(-.23, .22)$ \\
\hline DASS-Dep & 47 & $-.13(-.40, .16)$ & $.16(-.13, .42)$ & $.28(.00, .52) *$ & $-.03(-.31, .25)$ \\
DASS-Anx & 47 & $-.25(-.50, .03) \#$ & $.10(-.19, .37)$ & $.20(-.09, .46)$ & $-.06(-.33, .23)$ \\
DASS-Str & 47 & $-.16(-.42, .13)$ & $.18(-.11, .44)$ & $.31(.03, .54) *$ & $-.07(-.35, .21)$ \\
& & & & & \\
DASS-Tot & 47 & $-.19(-.44, .10)$ & $.19(-.09, .45)$ & $.27(-.01, .51)^{\#}$ & $-.10(-.37, .19)$ \\
\end{tabular}

Notes. ${ }^{*} \mathrm{p}<.05,{ }^{\#} \mathrm{p}<.10 ;$ STAI-T $=$ State Trait Anxiety Inventory, Trait scale; - Anx $=$ Set of items previously identified by (Bieling et al., 1998) to be primarily related to anxiety; -Dep = Set of items previously identified to be primarly related to depression; SDS = Self-rating Depression Scale; DASS = Depression, Anxiety, and Stress Scales.

Sample 2 (Clinical). As with Sample 1, there were no significant negative relationships between symptom measures and volatility-induced learning-rate adaptation nor was there a consistent pattern of associations over all (Table 3). Instead, we again observed a significant positive correlation between measures of anxiety/depression and learning-rate adaptation for negative outcomes; however, in Sample 2, this association occurred under conditions of rewardmaximisation, rather than punishment-minimisation.

\section{Discussion}

Inconsistent with prior work (Browning et al., 2015), we did not find evidence of a reliable negative association between volatility-induced learning-rate adaptation and anxiety, nor did we find a consistent pattern of associations across our two samples. Additionally, we did not find that significant relationships between learning and psychopathology were specific to anxiety. Instead, we found that relationships between volatility-induced learning-rate adaptation and symptom measures, where they were significant, were positive in direction, restricted to negative 
outcomes, and largely independent of distinctions between anxiety or depression. Discrepancies between the two samples relate to differences in performance across incentive conditions; while symptom measures were related to performance under conditions of punishment-minimisation and not reward-maximisation in the non-clinical convenience sample, the opposite was true for the clinical sample. Consistent with recent findings by Gagne and colleagues (2020), our results seem to suggest that individuals higher in negative affect, rather than depression or anxiety specifically, tend to more quickly adapt to changes in environmental volatility when responding to negative outcomes. Put another way, when uncertainty around the best course of action increases (i.e., the environment becomes more volatile), those high in negative affect tend to increase weight given to negative, rather than positive, outcomes when determining future behaviour.

The lack of a significant relationship between volatility-induced learning-rate adaptation and total trait anxiety in Sample 1 may indicate that related learning deficits are not as central to trait anxiety as previously suggested. While there was an association of trait anxiety with negative outcomes under punishment-minimisation $(r=0.19)$, it was smaller than in previous work $(r=-0.4)$. The effect of our volatility manipulation in Sample 1, as well as the levels of trait anxiety in our sample, were similar to those in prior work. Additionally, our sample size was roughly 2.5 times larger than work by Browning and colleagues (2015), though approximately half the size of work by Gagne and colleagues (2020).

Our results are inconsistent with those of prior work (Browning et al., 2015) and conflict with a wide literature linking symptoms of depression to blunted responding overall (Alloy et al., 2016; Huys et al., 2013), particularly under conditions of reward-maximisation (DelDonno et al., 2015; Herzallah et al., 2013). Despite inconsistencies with prior work, our results support the recent hypothesis proposed by Pulcu \& Browning (2019) and the findings of Gagne and 
colleagues (2020); perception/belief that negative outcomes are more informative of future outcomes than are positive outcomes may underpin emotional disorders. Our results are also consistent with literature linking symptoms of depression to improved performance on cognitive tasks under conditions of punishment-minimization (Beevers et al., 2013; Maddox et al., 2012) and persistent reliance on negative feedback even when it is uninformative (Murphy et al., 2003; Tavares et al., 2008). Additionally, our results fit with previous work into neuroticism (Chan et al., 2007) and tenants of established psychological treatments for affective disorders such as cognitive-behavioural therapy (Beck \& Haigh, 2014). These treatment methods broadly propose that pathological anxiety/depression arises from biased use of negatively-valenced information when appraising past, current, or future events and recommend various strategies for the correction of this bias (e.g., behavioural activation, structured reality testing, thought challenging, journals containing positive events).

\section{Replication implications}

We conceptually replicated prior work, leaving open the possibility that specific features of prior approaches, omitted or changed by us, are important to previously observed effects. The most obvious difference to prior work is the use of socio-affective feedback. Previous work has demonstrated that electrical shock is a more potent learning stimulus than affective faces paired with auditory screaming (Glenn et al., 2012). Another critical departure in our methods was the coding of socio-affective feedback as dichotomous outcomes. Continuous variables, if their measured magnitude corresponds to values used by participants in decision making, provide additional constraints upon model parameters. Consequently, it is possible that, by using a dichotomous rather than continuous feedback variable, the reliability of our parameter estimates was diminished. Low measurement reliability has consequences both at the group level (e.g., in studies such as ours) and at the level of the individual (e.g., when intending to use measures in 
clinical settings). With respect to current results, low reliability reduces the capacity to detect group level associations between behavioural and symptom measures, increasing the chance of a type II error.

\section{Clinical Implications}

A goal of the current study was to utilise the methods that we have employed to develop better behavioural assays of depression and anxiety (Hitchcock et al., 2017; Montague et al., 2012; Paulus et al., 2016), potentially identifying functional clinical subgroups based on meaningful behavioural deficits (Wiecki et al., 2015). While testing the efficacy of these techniques as clinical assays was not the purpose of this study, several components of our results, including issues with parameter reliability noted above, are suggestive of significant barriers to utilising these techniques within clinical settings.

One issue was the moderately poor rates of task comprehension and completion. Roughly $15 \%$ and $26 \%$ of participants were excluded due to poor task performance (i.e., their performance was best described as guessing) in the non-clinical and clinical samples respectively. Additionally, the hypothesised experimental effect of volatility on learning was not evident in the clinical sample, despite practice trials, a reasonably simple task structure, and numerous studies suggesting that adaptation to environmental volatility is a robust effect (Behrens et al., 2007, 2008; Browning et al., 2015; Pulcu \& Browning, 2017). One explanation for the above discrepancy relates to the composition of our sample relative to those in prior work (Browning et al., 2015), who excluded individuals undergoing treatment for a mental disorder, though others have included similar samples (see Gagne et al. 2020). Rates of poor task completion similar to our own were found by previous work using a clinical sample (Chase et al., 2010). Thus, issues with task comprehension/performance may be a problem inherent to working within ecologically valid clinical populations. 
A common factor underlying some issues listed above is the relatively low number of trials on which we based our estimates. More trials would (a) allow some to be sacrificed as practice, increasing the chance that individuals understand the task before commencing, and (b) increase the reliability of parameter estimates. With more reliable parameter estimates we would also be better situated to estimate the nature and size of any effects psychopathology may have on value-based decision-making. However, while the solution is simple, the implementation is not. Sessions of the task in the present study took approximately 15 minutes each; asking participants to concentrate for much longer in routine clinical settings may not be feasible since the number of trials tolerable to a clinical population is often less than is desirable for accurate parameter estimation. Operating within the limits of what is indicated for such a population (Rolstad et al., 2011), our own simulations (see Supplemental Material) suggest that parameter estimates are only moderately reliable at best. Such findings raise questions about the utility of current computational psychiatry measures in clinical settings, since low reliability renders individual measurements less interpretable.

Despite the limitations noted above, our study had a number of strengths. Through the use of socio-affective feedback, we were able to compare differential performance under shifting incentives without the confound of feedback modality. Additionally, through the application of computational models as measurement tools, we were able to describe relationships between learning processes and symptoms not evident at the level of composite behaviour. Our results support the view that behavioural abnormalities in anxiety and depression are dependent on the incentive structure of the environment and are best described using computational models able to parse behavioural data into variance associated with relevant component processes. 


\section{Concluding Remarks}

Our results add to the already substantial evidence supporting the role of abnormal valuebased decision-making in emotional disorders (Bishop \& Gagne, 2018; Paulus \& Yu, 2012; Robinson \& Chase, 2017). Through the formal modelling of behaviour in an operant learning task, we found (consistent with other recent work; Gagne et al. 2020) that general characteristics of depression and anxiety (e.g., negative affect) were related to increased reliance on information from negative outcomes under conditions of increased uncertainty. Additionally, by using socioaffective feedback as reinforcement stimuli, our work indicates that these effects are likely to be present in situations relevant for human functioning (e.g., social situations) and that interactions between incentive condition and other effects are unlikely to be solely a function of the modality of feedback (monetary gain/aversive shock). Finally, our results reinforce the need to (a) measure behaviour under conditions of both punishment-minimisation and reward-maximisation, (b) model learning related to positive and negative outcomes separately, and (c) assess symptoms of both anxiety and depression, insofar as these constructs are separable, when seeking to characterise the relationship between learning and emotional disorders. 


\section{References}

Adams, R. A., Huys, Q. J., \& Roiser, J. P. (2016). Computational psychiatry: Towards a mathematically informed understanding of mental illness. Journal of Neurology, Neurosurgery \& Psychiatry, 87(1), 53-63.

Ahn, W.-Y., Haines, N., \& Zhang, L. (2017). Revealing neurocomputational mechanisms of reinforcement learning and decision-making with the hBayesDM package. Computational Psychiatry, 1, 24-57.

Alloy, L. B., Olino, T., Freed, R. D., \& Nusslock, R. (2016). Role of reward sensitivity and processing in major depressive and bipolar spectrum disorders. Behavior Therapy, 47(5), $600-621$.

American Psychiatric Association. (2013). Diagnostic and statistical manual of mental disorders: DSM-5 (5th ed.). Author.

Andrews, G., \& Slade, T. (2001). Interpreting scores on the Kessler psychological distress scale (K10). Australian and New Zealand Journal of Public Health, 25(6), 494-497.

Antony, M. M., Bieling, P. J., Cox, B. J., Enns, M. W., \& Swinson, R. P. (1998). Psychometric properties of the 42-item and 21-item versions of the Depression Anxiety Stress Scales in clinical groups and a community sample. Psychological Assessment, 10(2), 176.

Beck, A. T., \& Haigh, E. A. P. (2014). Advances in Cognitive Theory and Therapy: The Generic Cognitive Model*. Annual Review of Clinical Psychology, 10, 1-24. https://doi.org/doi:10.1146/annurev-clinpsy-032813-153734 
Beevers, C. G., Worthy, D. A., Gorlick, M. A., Nix, B., Chotibut, T., \& Maddox, W. T. (2013). Influence of depression symptoms on history-independent reward and punishment processing. Psychiatry Research, 207(1-2), 53-60.

Behrens, T. E., Hunt, L. T., Woolrich, M. W., \& Rushworth, M. F. (2008). Associative learning of social value. Nature, 456(7219), 245.

Behrens, T. E., Woolrich, M. W., Walton, M. E., \& Rushworth, M. F. (2007). Learning the value of information in an uncertain world. Nature Neuroscience, 10(9), 1214.

Bieling, P. J., Antony, M. M., \& Swinson, R. P. (1998). The State-Trait Anxiety Inventory, Trait version: Structure and content re-examined. Behaviour Research and Therapy, 36(7-8), $777-788$.

Bishop, S. J., \& Gagne, C. (2018). Anxiety, depression, and decision making: A computational perspective. Annual Review of Neuroscience, 41, 371-388.

Bonett, D. G. (2008). Confidence intervals for standardized linear contrasts of means. Psychological Methods, 13(2), 99.

Brown, T. A., Chorpita, B. F., Korotitsch, W., \& Barlow, D. H. (1997). Psychometric properties of the Depression Anxiety Stress Scales (DASS) in clinical samples. Behaviour Research and Therapy, 35(1), 79-89.

Browning, M., Behrens, T. E., Jocham, G., O’Reilly, J. X., \& Bishop, S. J. (2015). Anxious individuals have difficulty learning the causal statistics of aversive environments. Nature Neuroscience, 18(4), 590. 
Carpenter, B., Gelman, A., Hoffman, M. D., Lee, D., Goodrich, B., Betancourt, M., Brubaker, M., Guo, J., Li, P., \& Riddell, A. (2017). Stan: A probabilistic programming language. Journal of Statistical Software, 76(1).

Chan, S. W., Goodwin, G. M., \& Harmer, C. J. (2007). Highly neurotic never-depressed students have negative biases in information processing. PSYCHOLOGICAL MEDICINELONDON-, 37(9), 1281.

Chase, H., Frank, M., Michael, A., Bullmore, E., Sahakian, B., \& Robbins, T. (2010). Approach and avoidance learning in patients with major depression and healthy controls: Relation to anhedonia. Psychological Medicine, 40(3), 433-440.

Clithero, J. A., \& Rangel, A. (2014). Informatic parcellation of the network involved in the computation of subjective value. Social Cognitive and Affective Neuroscience, 9(9), 1289-1302.

De Berker, A. O., Rutledge, R. B., Mathys, C., Marshall, L., Cross, G. F., Dolan, R. J., \& Bestmann, S. (2016). Computations of uncertainty mediate acute stress responses in humans. Nature Communications, 7, 10996.

DelDonno, S. R., Weldon, A. L., Crane, N. A., Passarotti, A. M., Pruitt, P. J., Gabriel, L. B., Yau, W., Meyers, K. K., Hsu, D. T., Taylor, S. F., \& others. (2015). Affective personality predictors of disrupted reward learning and pursuit in major depressive disorder. Psychiatry Research, 230(1), 56-64.

Denwood, M. J. (2016). runjags: An R package providing interface utilities, model templates, parallel computing methods and additional distributions for MCMC models in JAGS. Journal of Statistical Software, 71(9), 1-25. 
Gagne, C., Zika, O., Dayan, P., \& Bishop, S. J. (2020). Impaired adaptation of learning to contingency volatility in internalizing psychopathology. ELife, 9, e61387. https://doi.org/10.7554/eLife.61387

Glenn, C. R., Lieberman, L., \& Hajcak, G. (2012). Comparing electric shock and a fearful screaming face as unconditioned stimuli for fear learning. International Journal of Psychophysiology, 86(3), 214-219.

Herzallah, M. M., Moustafa, A. A., Natsheh, J. Y., Abdellatif, S. M., Taha, M. B., Tayem, Y. I., Sehwail, M. A., Amleh, I., Petrides, G., Myers, C. E., \& others. (2013). Learning from negative feedback in patients with major depressive disorder is attenuated by SSRI antidepressants. Frontiers in Integrative Neuroscience, 7, 67.

Hishinuma, E. S., Miyamoto, R. H., Nishimura, S. T., Goebert, D. A., Yuen, N. Y., Makini Jr, G. K., Andrade, N. N., Johnson, R. C., \& Carlton, B. S. (2001). Prediction of anxiety disorders using the state-trait anxiety inventory for multiethnic adolescents. Journal of Anxiety Disorders, 15(6), 511-533.

Hitchcock, P., Niv, Y., Radulescu, A., \& Sims, C. R. (2017). Translating a Reinforcement Learning Task into a Computational Psychiatry Assay: Challenges and Strategies. In CogSci.

Huys, Q. J., Pizzagalli, D. A., Bogdan, R., \& Dayan, P. (2013). Mapping anhedonia onto reinforcement learning: A behavioural meta-analysis. Biology of Mood \& Anxiety Disorders, 3(1), 12.

Kessler, R. C., Andrews, G., Colpe, L. J., Hiripi, E., Mroczek, D. K., Normand, S.-L., Walters, E. E., \& Zaslavsky, A. M. (2002). Short screening scales to monitor population prevalences 
and trends in non-specific psychological distress. Psychological Medicine, 32(6), 959976.

Knight, R. G., Waal-Manning, H. J., \& Spears, G. F. (1983). Some norms and reliability data for the State-Trait Anxiety Inventory and the Zung Self-Rating Depression scale. British Journal of Clinical Psychology, 22(4), 245-249.

Lecrubier, Y., Sheehan, D. V., Weiller, E., Amorim, P., Bonora, I., Sheehan, K. H., Janavs, J., \& Dunbar, G. C. (1997). The Mini International Neuropsychiatric Interview (MINI). A short diagnostic structured interview: Reliability and validity according to the CIDI. European Psychiatry, 12(5), 224-231.

Liu, X., Ma, D., Kurita, H., \& Tang, M. (1999). Self-reported depressive symptoms among Chinese adolescents. Social Psychiatry and Psychiatric Epidemiology, 34(1), 44-47.

Lovibond, P. F., \& Lovibond, S. H. (1995). The structure of negative emotional states: Comparison of the Depression Anxiety Stress Scales (DASS) with the Beck Depression and Anxiety Inventories. Behaviour Research and Therapy, 33(3), 335-343.

Lovibond, S. H., \& Lovibond, P. F. (1996). Manual for the depression anxiety stress scales. Psychology Foundation of Australia.

Maddox, W. T., Gorlick, M. A., Worthy, D. A., \& Beevers, C. G. (2012). Depressive symptoms enhance loss-minimization, but attenuate gain-maximization in history-dependent decision-making. Cognition, 125(1), 118-124.

Montague, P. R., Dolan, R. J., Friston, K. J., \& Dayan, P. (2012). Computational psychiatry. Trends in Cognitive Sciences, 16(1), 72-80. 
Murphy, F., Michael, A., Robbins, T., \& Sahakian, B. (2003). Neuropsychological impairment in patients with major depressive disorder: The effects of feedback on task performance. Psychological Medicine, 33(3), 455-467.

Pauli, W. M., Larsen, T., Collette, S., Tyszka, J. M., Seymour, B., \& O’Doherty, J. P. (2015). Distinct Contributions of Ventromedial and Dorsolateral Subregions of the Human Substantia Nigra to Appetitive and Aversive Learning. Journal of Neuroscience, 35(42), 14220-14233. https://doi.org/10.1523/JNEUROSCI.2277-15.2015

Paulus, M. P., Huys, Q. J., \& Maia, T. V. (2016). A roadmap for the development of applied computational psychiatry. Biological Psychiatry: Cognitive Neuroscience and Neuroimaging, 1(5), 386-392.

Paulus, M. P., \& Yu, A. J. (2012). Emotion and decision-making: Affect-driven belief systems in anxiety and depression. Trends in Cognitive Sciences, 16(9), 476-483.

Peirce, J., Gray, J. R., Simpson, S., MacAskill, M., Höchenberger, R., Sogo, H., Kastman, E., \& Lindeløv, J. K. (2019). PsychoPy2: Experiments in behavior made easy. Behavior Research Methods, 51(1), 195-203.

Pulcu, E., \& Browning, M. (2017). Affective bias as a rational response to the statistics of rewards and punishments. Elife, 6, e27879.

Pulcu, E., \& Browning, M. (2019). The misestimation of uncertainty in affective disorders. Trends in Cognitive Sciences, 23(10), 865-875.

Regier, D. A., Narrow, W. E., Clarke, D. E., Kraemer, H. C., Kuramoto, S. J., Kuhl, E. A., \& Kupfer, D. J. (2013). DSM-5 field trials in the United States and Canada, Part II: testretest reliability of selected categorical diagnoses. American Journal of Psychiatry, $170(1), 59-70$. 
Robins, L. N., Wing, J., Wittchen, H. U., Helzer, J. E., Babor, T. F., Burke, J., Farmer, A., Jablenski, A., Pickens, R., Regier, D. A., \& others. (1988). The Composite International Diagnostic Interview: An epidemiologic instrument suitable for use in conjunction with different diagnostic systems and in different cultures. Archives of General Psychiatry, 45(12), 1069-1077.

Robinson, O. J., \& Chase, H. W. (2017). Learning and choice in mood disorders: Searching for the computational parameters of anhedonia. Computational Psychiatry, 1, 208-233.

Rolstad, S., Adler, J., \& Rydén, A. (2011). Response Burden and Questionnaire Length: Is Shorter Better? A Review and Meta-analysis. Value in Health, 14(8), 1101-1108. https://doi.org/10.1016/j.jval.2011.06.003

Sheehan, D. V., Lecrubier, Y., Sheehan, K. H., Amorim, P., Janavs, J., Weiller, E., Hergueta, T., Baker, R., \& Dunbar, G. C. (1998). The Mini-International Neuropsychiatric Interview (MINI): The development and validation of a structured diagnostic psychiatric interview for DSM-IV and ICD-10. The Journal of Clinical Psychiatry.

Shek, D. T. (1988). Reliability and factorial structure of the Chinese version of the State-Trait Anxiety Inventory. Journal of Psychopathology and Behavioral Assessment, 10(4), 303317.

Shek, D. T. (1993). The Chinese version of the State-Trait Anxiety Inventory: Its relationship to different measures of psychological well-being. In Journal of clinical psychology (Vol. 49, Issue 3, pp. 349-358).

Spielberger, C. D., Gorsuch, R. L., Lushene, R., Vagg, P., \& Jacobs, G. (1983). Consulting Psychologists Press; Palo Alto, CA: 1983. Manual for the State-Trait Anxiety Inventory. 
Spitzer, R., Williams, J., Gibbon, M., \& First, M. (1988). Structured Clinical Interview for DSMIII-R-Patient Version. New York: New York State Psychiatric Institute. Biometrics Research Department.

Tavares, J. V. T., Clark, L., Furey, M. L., Williams, G. B., Sahakian, B. J., \& Drevets, W. C. (2008). Neural basis of abnormal response to negative feedback in unmedicated mood disorders. Neuroimage, 42(3), 1118-1126.

Team, R. C. (2013). R: A language and environment for statistical computing. https://www.Rproject.org/

Thoma, P., Bauser, D. S., \& Suchan, B. (2013). BESST (Bochum Emotional Stimulus Set)—A pilot validation study of a stimulus set containing emotional bodies and faces from frontal and averted views. Psychiatry Research, 209(1), 98-109.

Thurber, S., Snow, M., \& Honts, C. R. (2002). The Zung self-rating depression scale: Convergent validity and diagnostic discrimination. Assessment, 9(4), 401-405.

Vehtari, A., Gelman, A., \& Gabry, J. (2017). Practical Bayesian model evaluation using leaveone-out cross-validation and WAIC. Statistics and Computing, 27(5), 1413-1432.

Watson, D. (2009). Differentiating the mood and anxiety disorders: A quadripartite model. Annual Review of Clinical Psychology, 5, 221-247.

Wiecki, T. V., Poland, J., \& Frank, M. J. (2015). Model-based cognitive neuroscience approaches to computational psychiatry: Clustering and classification. Clinical Psychological Science, 3(3), 378-399.

Zung, W. W. (1965). A self-rating depression scale. Archives of General Psychiatry, 12(1), 6370. 
